\title{
Elastic energy loss and longitudinal straggling of a hard jet
}

\author{
A. Majumder \\ Department of Physics, Duke University, Durham, North Carolina 27708, USA and \\ Department of Physics, Ohio State University, Columbus, Ohio 43210, USA
}

(Received 22 December 2008; published 23 September 2009)

\begin{abstract}
The elastic energy loss encountered by jets produced in deep-inelastic scattering (DIS) off a large nucleus is studied in the collinear limit. In close analogy to the case of (nonradiative) transverse momentum broadening, which is dependent on the medium transport coefficient $\hat{q}$, a class of medium enhanced higher twist operators which contribute to the nonradiative loss of the forward light-cone momentum of the jet $\left(q^{-}\right)$are identified and the leading correction in the limit of asymptotically high $q^{-}$is isolated. Based on these operator products, a new transport coefficient $\hat{e}$ is motivated which quantifies the energy loss per unit length encountered by the hard jet. These operator products are then computed, explicitly, in the case of a similar hard jet traversing a deconfined quark-gluon plasma (QGP) in the hard-thermal-loop (HTL) approximation. This is followed by an evaluation of subleading contributions which are suppressed by the inverse light-cone momentum $q^{-}$, which yields the longitudinal "straggling," i.e., a slight change in light cone momentum due to the Brownian propagation through a medium with a fluctuating color field.
\end{abstract}

DOI: 10.1103/PhysRevC.80.031902

PACS number(s): 12.38.Mh, 11.10.Wx, 25.75.Dw

The modification of hard jets as they propagate through dense matter is now a rather sophisticated enterprise [1-5], encompassing the study of jets propagating through cold nuclear matter in deep-inelastic scattering off large nuclei [6] as well as jets in hot deconfined matter produced in the collision of heavy-ions at high energy [7]. Unlike most approaches to jet modification, the higher-twist expansion approach [3] attempts to decompose the measured modification into a part computable using perturbative QCD (pQCD) and a set of nonperturbative "jet-transport coefficients" which are then used to quantify the properties of the dense matter. Since very few assumptions about the medium have been made, this formalism is equally applicable to both confined and deconfined matter. As a result, such a study has universal applications to a variety of heavy-ion experiments at the Relativistic Heavy-Ion Collider (RHIC) and the Large Hadron Collider (LHC) and to DIS experiments at the Continuous Electron Beam Accelerator Facility (CEBAF) and the future Electron-Ion Collider (EIC). To date, most jet modification calculations, in the higher twist scheme, have been limited to radiative energy loss and transverse broadening of the hard jet [8]. Both these signals of jet modification are dependent on the transverse transport parameter $\hat{q}$ : which encodes the mean transverse momentum squared picked up by a hard jet per unit length of medium traversed [1].

In this Rapid Communication, the longitudinal momentum loss of the jet due to elastic exchange with the medium will be explored. We begin by first defining and clarifying the notion of elastic energy loss. It should be pointed out, that this is by far not the first attempt to explore this avenue of jet modification (see for instance Refs. [9-12]). However, most of these computations assume a specific model of the medium: that of a thermalized plasma of quarks and gluons both within the HTL limit (as in Refs. [9,10,12]) and without invoking such limits [11]. Our formulation, in this manuscript, assumes no particular model of the medium and is thus related to that of Ref. [11]. However, our results will differ from those of
Ref. [11] in that we identify a process which does not interfere with radiative energy loss and depends on a new transport coefficient. In so doing, the manuscript strives to attain a nonambiguous definition of elastic energy loss within the various scenarios that may be encountered in current experiments on DIS and heavy-ion collisions.

It is no surprise that the magnitude of elastic energy loss (and even radiative energy loss) is a frame dependent statement. While in heavy-ion collisions, the natural frame of choice is the center-of-mass frame of the two colliding heavy ions, there is more than one obvious choice in DIS on a large nucleus. As a result, in this Rapid Communication, the focus will lie on the approximately boost invariant quantity of fractional light-cone momentum loss,

$$
z=\frac{E_{\text {lost }}+p_{\text {lost }}^{3}}{E+p^{3}}
$$

where $E_{\text {lost }}$ and $p_{\text {lost }}^{3}$ refer to the energy and $z$ component of the momentum lost by a hard parton with energy and $z$ component of the momentum given by $E$ and $p^{3}$. The qualifier, approximately boost invariant, is used as the above quantity is only invariant for the case of boosts restricted solely to the three-direction, or the direction of the chosen component of momentum. Such a construction is somewhat alien to treatments of elastic energy loss in classical electrodynamics (see Chap. 13 of Ref. [13]), where the lost momentum is usually transverse to the direction of motion of the fast moving charge.

Imagine that a hard quark is produced in the DIS of an electron on a large nucleus. Such an analysis is carried out with maximal ease in the Breit frame, where both the virtual photon and the large nucleus approach each other at a large momentum [14]. In the interest of simplicity, we imagine that the nucleus moves in the positive $z$ direction and has a large momentum $A p^{+}$, where $A$ is the atomic number of the nucleus. A hard quark with light cone momentum $x_{B} p^{+}$is struck by the 
virtual photon which moves in the negative $z$ direction with momentum

$$
q_{\gamma}=\left[-x_{B} p^{+}, q^{-}, 0,0\right],
$$

where, the Björken variable has the usual value $x_{B}=$ $Q^{2} /\left(2 p^{+} q^{-}\right)$in terms of the virtuality $Q^{2}$ of the virtual photon. In this frame, the struck quark has its four-momentum changed to $l=x_{B} p+q \simeq\left[0, q^{-}, 0,0\right]$, may be thought of as close to on-shell and moving in a direction opposite to that of the large nucleus. The differential hadronic tensor for the production of an on-shell hard quark with a momentum $l$ in the interaction of a virtual photon on a large nucleus $A$ is given as

$$
\frac{d^{3} W_{0}^{\mu \nu}}{d^{2} l_{\perp} d l^{-}}=W_{0}^{\mu v} \delta^{2}\left(\vec{l}_{\perp}\right) \delta\left(l^{-}-q^{-}\right),
$$

where $W_{0}^{\mu \nu}$ represents the inclusive hadronic tensor at leading order and leading twist, i.e.,

$$
W_{0}^{\mu \nu}=-C_{p}^{A} 2 \pi g_{\perp}^{\mu \nu} \sum_{q} Q_{q}^{2} f_{q}\left(x_{B}\right),
$$

where, we have used the light-cone notation $-g_{\perp}^{\mu \nu}=$ $g^{\mu+} g^{\nu-}+g^{\nu+} g^{\mu-}-g^{\mu \nu}$. The coefficient $C_{p}^{A}$ simply counts the number of nucleons in the nucleus that the jet may scatter off and is equal to $A$.

In the remainder, we focus on the multiple soft reinteractions of this quark with the soft glue field within the various nucleons. The weakly interacting picture of nucleons in the large nucleus (which is approximately valid at very high energies) is now imposed (see Ref. [14] for details). Interactions of the quark with the various nucleons may henceforth be considered to be independent and thus uncorrelated. This step assumes a factorization of the production process of the hard quark and its later soft scattering off the glue field. As a result, a reader, more interested in the interactions of a quark with deconfined matter, may replace the individual nucleons with the prevalent degrees of freedom of the matter under study $[15,16]$.

Consider the soft rescattering of the hard quark with one such nucleon (or degree of freedom). In this limit of factorized interactions of the hard quark with the various nucleons, the effect of multiple interactions on the propagation of the hard quark may be iterated from the effect of a single interaction. This is not true in general and arguments will be forwarded which justify the use of this scheme in this particular case. By the effect on the hard quark, we specifically mean the effect on its three dimensional distribution in momentum space, i.e., in terms of $l^{-}, \vec{l}_{\perp}$. The aim is to identify the form of the distribution $\phi_{L}\left(l^{-}, \vec{l}_{\perp}\right)$, after the parton has traversed a certain length $L$ given an initial distribution,

$$
\phi_{0}\left(l^{-}, \vec{l}_{\perp}\right)=\delta^{2}\left(\vec{l}_{\perp}\right) \delta\left(l^{-}-q^{-}\right) .
$$

The hadronic tensor with two gluon scatterings in the final state, after a few simplifications, may be expressed as

$$
\begin{aligned}
W^{\mu \nu}= & \left(-g_{\perp}^{\mu \nu}\right) C_{p, p_{2}}^{A} \int d y_{0}^{-} e^{-i x_{B} p^{+} y_{0}^{-}}\left\langle p\left|\bar{\psi}\left(y_{0}^{-}\right) \frac{\gamma^{+}}{2} \psi(0)\right| p\right\rangle \\
& \times g^{2} \int \frac{d l^{-} d^{2} l_{\perp}}{(2 \pi)^{3}} d Y^{-} d y^{-} d y^{+} d^{2} y_{\perp} \frac{d k^{-} d^{2} k_{\perp}}{(2 \pi)^{3}}
\end{aligned}
$$

$$
\begin{aligned}
& \times \frac{(2 \pi)^{3} \delta\left(l^{-}-q^{-}-k^{-}\right) \delta^{2}\left(\vec{l}_{\perp}-\vec{k}_{\perp}\right)}{2\left(q^{-}+k^{-}\right)} \frac{\operatorname{Tr}\left[t^{a} t^{b}\right]}{N_{c}} \\
& \times \frac{\operatorname{Tr}}{4}\left[\gamma^{+} \gamma^{\alpha}\left\{\left(q^{-}+k^{-}\right)+\frac{\gamma^{-} k_{\perp}^{2}}{2\left(q^{-}+k^{-}\right)}-k_{\perp}\right\} \gamma^{\beta}\right] \\
& \times \exp \left[-i \frac{k_{\perp}^{2}}{2 q^{-}}\left(y^{-}\right)+i y_{\perp} \cdot k_{\perp}-i y^{+} k^{-}\right] \\
& \times\left\langle p_{2}\left|A_{\alpha}^{a}\left(Y^{-}+y\right) A_{\beta}^{b}\left(Y^{-}\right)\right| p_{2}\right\rangle .
\end{aligned}
$$

In the limit of large energy, $q^{-} \rightarrow \infty$, the distribution of $k^{-}$ which is obtained by Fourier transforming the $y^{+}$dependence of the product $A^{\alpha}\left(Y^{-}+y\right) A^{\beta}\left(Y^{-}\right)$will be dominated by values of $k^{-} \ll q^{-}$. Under these limits, $k^{-}$may be dropped from the momentum dependent part of the integrand [third and fourth line of Eq. (6)] and the integral over $k^{-}$may be performed using the phase factor $\exp \left(-i k^{-} y^{+}\right)$which constrains the entire process to the negative light cone. The resulting expression is then identical to the well known expression obtained for the standard treatment of transverse broadening with scattering off a single gluon in the final state [17]. The coefficient $C_{p, p_{2}}^{A}$, where $|p\rangle$ represents the nucleon with the struck quark and $\left|p_{2}\right\rangle$ the nucleon which contains the soft gluons, accounts for $A$ times the weak correlation between the two nucleons involved. This coefficient is derived in Ref. [18] and simplifications discussed in Ref. [14].

In the evaluation of transverse broadening in Ref. [14], the two dimensional delta function $\delta^{2}\left(\vec{l}_{\perp}-\vec{k}_{\perp}\right)$ was expanded in a Taylor series in $k_{\perp}$ and the coefficients of each term of the expansion became the gluon matrix elements which appear in a product with the delta function. In the present case of nonradiative energy loss, we will follow a similar methodology and expand the delta function $\delta\left(l^{-}-q^{-}-k^{-}\right)$as a series in $k^{-}$and focus on the first derivative or the linear change in the distribution of $l^{-}$. Hence, the delta function may now be re-expressed as

$$
\begin{aligned}
\delta\left(l^{-}-q^{-}-k^{-}\right)= & \delta\left(l^{-}-q^{-}\right)-\frac{\partial \delta\left(l^{-}-q^{-}\right)}{\partial l^{-}}\left(k^{-}\right) \\
& +\frac{1}{2} \frac{\partial^{2}}{\partial l^{-2}} \delta\left(l^{-}-q^{-}\right)\left[k^{-}\right]^{2}+\cdots
\end{aligned}
$$

We use the equation above as a substitution for the $\delta$ function in Eq. (6) and identify the coefficient $\left(C_{1}\right)$ of the first derivative of the $\delta$ function as the magnitude of the collisional energy loss. This may be schematically decomposed as

$$
C_{1}=\mathcal{K}(k) \exp (k \cdot y)\langle\mathcal{Y}(y)\rangle
$$

i.e., a convolution of a purely momentum dependent part $\mathcal{K}$ with the expectation of a purely position dependent piece $\mathcal{Y}$ through a phase factor involving both.

In the limit of $\left|k^{-}\right| \ll q^{-}$and small coupling constant $g$, the momentum dependent piece $\mathcal{K}$ may be expanded in a series in $k^{-} / q^{-}$, i.e.,

$$
\begin{aligned}
\mathcal{K} \simeq & -k^{-}\left[q^{-} g^{\alpha+} g^{\beta+}+\frac{1}{2}\left(1-\frac{k^{-}}{q^{-}}\right)\right. \\
& \left.\times\left\{g^{\alpha+} k_{\perp}^{\beta}+g^{\beta+} k_{\perp}^{\alpha}\right\}-\frac{k_{\perp}^{2}}{4 q^{-}}\left(1-\frac{k^{-}}{q^{-}}\right) g_{\perp}^{\alpha \beta}\right] .
\end{aligned}
$$


The piece which depends on the coordinates may be simply expressed as $\mathcal{Y} \simeq A^{a \alpha}(y) A^{b^{\beta}}(0)$. In this expression, $y \equiv$ $\left(y^{+}, y^{-}, y_{\perp}\right)$ and assuming a weak dependence on $Y^{-}$, we have replaced $Y^{-}$with 0 simply to save writing. Integrating over $y^{+}$by parts, the overall factor of $k^{-}$in $\mathcal{K}$ is converted into the derivative $-i \partial^{-}$acting on $\mathcal{Y}$. The terms in $\mathcal{K}$ are then ordered in powers of $q^{-}$, the forward energy of the jet, which represents the largest scale in the problem. We begin by analyzing the leading term, which yields the coefficient of the first derivative of the longitudinal momentum $\delta$ function as

$$
\begin{aligned}
C_{1} \propto & \int d Y^{-} d^{4} y \frac{d k^{-} d^{2} k_{\perp}}{(2 \pi)^{3}} e^{-i \frac{k_{\perp}^{2}}{2 q^{-}} y^{-}-i k^{-} y^{+}+i k_{\perp} \cdot y_{\perp}} \\
& \times \frac{4 \pi \alpha}{2 N_{c}}\left\langle p_{2}\left|\left[i \partial^{-} A^{a+}(y) A^{a+}(0)\right]\right| p_{2}\right\rangle .
\end{aligned}
$$

The ' $\propto$ ' sign denotes that there is an over all factor that will arise from the decomposition of $C_{p, p_{2}}^{A}$ in Eq. (6) (see Refs. [14,18] for details). The coefficient $\left(C_{1}\right)$ above may be further simplified by ignoring the small factor $k_{\perp}^{2} /\left(2 q^{-}\right)$in the exponent and integrating out the $k_{\perp}$ and the $k^{-}$to yield $\delta$ functions over $y^{+}$and $y_{\perp}$.

Unlike the case of transverse broadening [14], the two gluon matrix element is not manifestly gauge invariant. It may be cast in a gauge covariant form by noting that the gauge field at $y^{+} \rightarrow \infty$ is vanishingly small. In this limit,

$$
\begin{aligned}
A^{a+}\left(Y^{-}\right) & =\int_{-\infty}^{0^{+}} d z^{+} \partial^{-} A^{a+}\left(Y^{-}, z^{+}\right) \\
& =\int_{-\infty}^{0} d z^{+} F^{a-+}\left(Y^{-}, z^{+}\right)
\end{aligned}
$$

This yields a somewhat complicated expression for the elastic energy loss coefficient. In the limit of $q^{-} \rightarrow \infty$, where one may ignore the $k_{\perp}^{2} /\left(2 q^{-}\right)$in the exponent, the coefficient is given as

$$
\begin{aligned}
C_{1}= & \int d Y^{-} d y^{-} d y^{+} \frac{4 \pi \alpha}{2 N_{c}} \frac{\rho_{N}}{2 p^{+}} \\
& \times\left\langle p_{2}\left|\left[i F^{a-+}\left(y^{-}, 0,0\right) F^{a-+}\left(0, y^{+}, 0\right)\right]\right| p_{2}\right\rangle .
\end{aligned}
$$

The factor of $\rho_{N} /\left(2 p^{+}\right)$, where, $\rho_{N}$ is the nucleon density and $p^{+}$is the average forward momentum of the nucleon, is obtained from a factorization of the correlation coefficient $C_{p, p_{2}}^{A} \simeq C_{p}^{A} \times \rho_{N} /\left(2 p^{+}\right)$, which is similar to the procedure used in Ref. [14] to indicate the weak correlation between nucleons.

In the derivation of the leading contribution to elastic energy loss in large nuclei, Eq. (12) is as far as one may proceed without invoking a model of the gluon distribution within the nucleons. In the case of transverse broadening [14], a similar distribution involving the gauge field $F^{+\perp}$ results; this is combined with the nucleon density $\rho_{N}$ to define the transport coefficient $\hat{q}$. In transverse broadening and energy loss in both large nuclei and heavy-ion collisions, $\hat{q}$ is often used as a parameter to fit with experimental data [19]. We anticipate that a similar coefficient may also be motivated for the elastic energy loss, denoted as

$$
\begin{aligned}
\hat{e}= & \frac{4 \pi \alpha}{2 N_{c}} \frac{\rho}{2 p^{+}} \\
& \times \int d y^{-} d y^{+}\left\langle p\left|\left[i F^{a-+}\left(y^{-}, 0,0\right) F^{a-+}\left(0, y^{+}, 0\right)\right]\right| p\right\rangle .
\end{aligned}
$$

The coefficient above represents the leading contribution to the elastic energy loss per unit length as encountered by a hard jet due to soft rescattering in the medium. There exist multiple other contributions such as energy loss due to hard rescattering, Compton scattering off hard gluons and subleading contributions (in $q^{-}$power counting) from Eq. (9). The first two contributions were calculated in Ref. [11] and the emission of a hard forward parton in the final state leads to an interference of such contributions with radiative energy loss. As a result, these contributions are appropriately combined with and should be considered as a part of the radiative energy loss calculation [3]. In this manuscript, the focus has been restricted only on those subleading contributions which do not have a hard on-shell gluon in the final state and thus do not interfere with radiative energy loss. Alternatively stated, we are only considering contributions where the parton loses longitudinal momentum $q^{-}$without producing another hard parton with large (-)-component of light cone momentum.

As mentioned above, the effect of energy loss due to soft scattering off the glue field has been completely factorized from the production process of the hard jet. As a result, the coefficient $\hat{e}$ may be computed in any medium. In the case of elastic energy loss in a high temperature plasma, the coefficient in the equation above may be calculated exactly. Following the methods outlined in Ref. [14], we reabsorb the factor $\rho /\left(2 p^{+}\right)$ into the definition of a generalized medium state $|n\rangle$ and define the elastic loss coefficient as the expectation of the operator $i \partial^{-} A^{+}\left(y^{-}\right) A^{+}(0)$ in the ensemble of thermal states as

$$
\hat{e}_{\mathrm{HTL}}=\frac{4 \pi \alpha \int d y^{-}\left\langle n\left|e^{-\beta \hat{H}}\left[i \partial^{-} A^{a+}\left(y^{-}\right) A^{a+}(0)\right]\right| n\right\rangle}{2 N_{c}} .
$$

Using the standard decomposition of two-point operators in finite temperature field theory [20], we decompose the correlator in the equation above, in the HTL limit, as

$$
\begin{aligned}
\hat{e}_{\mathrm{HTL}}= & \frac{4 \pi \alpha_{s}}{2 N_{c}} \int \frac{d y^{-} d^{4} k}{(2 \pi)^{4}} e^{-i k^{+} y^{-}} \\
& \times k^{-} \rho_{a b}^{++}\left[1+n_{B}\left(k^{0}\right)\right] \delta^{a b},
\end{aligned}
$$

where $n_{B}$ is the Bose distribution function and $\rho_{a b}^{++}$represents the $(++)$-component of the gluon spectral density which is diagonal in color space $\left(\rho_{a b}=\rho \delta_{a b}\right)$.

The spectral density has the usual decomposition in terms of transverse and longitudinal components (using covariant gauge):

$$
\rho^{++}=P_{T}^{++} \rho_{T}+P_{L}^{++} \rho_{L}
$$

The projectors are further simplified on performing the $y^{-}$ integral which yields $\delta\left(k^{+}\right)$. As a result, $k^{-}=-2 k^{3}$ and $P_{T}^{++}=P_{T}^{33}=-P_{L}^{33}=\left|k_{\perp}^{2}\right| /\left|\left(k^{3}\right)^{2}+k_{\perp}^{2}\right|$. Using the standard notation $\left(k^{0} /|\vec{k}|=x\right)[20]$, the expression for the elastic energy 
loss per unit length is given as

$$
\begin{aligned}
\hat{e}= & 4 \pi \alpha_{s} \int_{0}^{Q_{\operatorname{MAX}}^{2}} \frac{d|\vec{k}|^{2}}{2 \pi} \int_{0}^{1} \frac{d x}{2 \pi} \frac{|\vec{k}|^{2}(-x)\left(1-x^{2}\right)\left(N_{c}^{2}-1\right)}{4 N_{c}} \\
& \times\left[\left\{\rho_{T}\left(|\vec{k}|, k^{0}\right)-\rho_{L}\left(|\vec{k}|, k^{0}\right)\right\}\left\{1+n_{B}\left(k^{0}\right)\right\}\right]_{k^{0}=-|\vec{k}| x} .
\end{aligned}
$$

The equation above for the loss of light cone momentum $l^{-}$per unit of light cone path $L^{-}$is equivalent to the energy lost by near on-shell partons per unit length, i.e.., $\hat{e} \simeq d E / d L$. It may be evaluated numerically using the actual form of the gluon spectral density. In realistic calculations of the elastic energy loss at finite temperature, the form of the spectral density depends on the exchanged momentum of the gluon. For hard exchanges, one may simply evaluate the $t$-channel matrix elements for the scattering of a hard jet parton off a hard $(\sim T)$ medium parton. These are logarithmically divergent and are regulated at a soft scale $\mu_{T}^{2}$. Soft exchanges below this scale are appropriately calculated within the HTL formalism. On combining both these computations the arbitrary scale $\mu_{T}^{2}$ is removed (see Refs. [9,12] for details and results of this procedure).

The goal of this Rapid Communication is not to rederive the results of Refs. $[9,12]$ in their entirety but simply to show that a similar procedure may be carried out here with identical results. Adjusting for the choice of gauge and removing the color factor $C_{F}$ for the case of QED, Eq. (15) may be shown to be equivalent to Eq. (38) of the last article in Ref. [9]. For the case of soft momentum transfers, one may use the known form of the HTL spectral densities [20] to compute $\hat{e}$. Within this approximation, the upper limit of the $|\vec{k}|$ integral is terminated at an appropriate scale of $Q_{\mathrm{MAX}} \sim T$. With a choice of an $\alpha_{s}=0.3$ and a Debye mass of $m_{D} \simeq 4 \pi \alpha_{S} T$, this yields an $\hat{e} \sim 0.06 \mathrm{GeV} / \mathrm{fm}$ at a $T=300 \mathrm{MeV}$. In Refs. [9,12], the spectral density is rederived for the case of hard momentum transfers; the difference between the results obtained from such a procedure and that from arbitrarily extending the HTL form of the spectral density to large momentum transfers is small ( 20\%) at large jet energy $E(\sim 40-50 \mathrm{GeV})$ [21]. In this effort, we present this simplified estimate of the total elastic energy loss in a thermalized QCD medium by extending $Q_{\text {MAX }} \sim$ $\sqrt{E T}$. This yields an energy dependent elastic energy loss per unit length as shown in Fig. 1. The plot includes three different choices of $T$, and the coupling $\alpha_{s}$. The largest temperature and coupling yields the largest energy loss per unit length $(\hat{e})$, with $\hat{e}$ dropping when the temperature or the coupling is reduced.

Previous efforts using the higher twist formalism ignored this kind of mechanism of elastic energy loss. The current manuscript justifies the inclusion of such contributions within the basic formalism of higher twist elastic energy loss. Unlike the case of transverse broadening, there does not seem to be a simple classical analog of this calculation, however, attempts in this direction are currently underway [22].

A closely related calculation, at the same order of expansion in $\alpha_{s}$, is the fluctuation of the elastic energy loss. This is obtained as the coefficient of the second derivative of the delta function of Eq. (7). Following a procedure almost identical to the steps carried out above, we obtain this coefficient in a

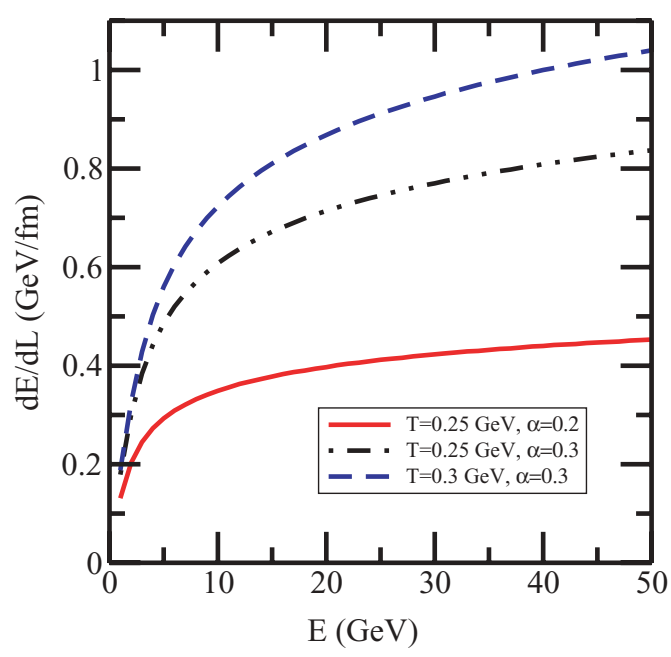

FIG. 1. (Color online) Elastic energy loss per unit length $(d E / d L)$ as a function of the energy of the propagating parton for different fixed temperatures of the media and values of $\alpha_{s}$. See text for details.

thermal medium as

$$
\hat{e}_{2}=\frac{4 \pi \alpha \int d y^{-}\left\langle n\left|e^{-\beta \hat{H}}\left[F^{a-+} F^{a-+}\right]\right| n\right\rangle}{2 N_{c}},
$$

where $F^{a-+}$ has been defined in Eq. (11). Since there is no assumption of equilibrium in the higher twist formalism, one cannot relate the transport coefficients $\hat{e}$ and $\hat{e}_{2}$ by a fluctuation-dissipation theorem. Any such relation will require additional conditions not in this current formalism. Following a resummation procedure, as in Ref. [14], and by assuming that the correlation between $F^{+\perp}$ and $F^{+-}$is vanishing, one may immediately postulate the diffusion equation for elastic energy loss as

$$
\frac{\partial \phi\left(L^{-}, l^{-}\right)}{\partial L^{-}}=\hat{e} \frac{\partial \phi\left(L^{-}, l^{-}\right)}{\partial l^{-}}+\hat{e}_{2} \frac{\partial^{2} \phi\left(L^{-}, l^{-}\right)}{\partial\left(l^{-}\right)^{2}},
$$

where $\hat{e}$ and $\hat{e}_{2}$ are the diffusion and drag coefficients.

In the interest of completeness, we will also evaluate the next-to-leading terms in Eq. (9) which provide additional contributions to the elastic energy loss. These are however, further suppressed by the hard scale of $q^{-}$. The next-to-leading contribution to the elastic energy loss or expectation of $k^{-}$ emanates from the contraction of the second and term in $\mathcal{K}$ with $\mathcal{Y}$ from Eq. (9). Evaluating this contribution, we obtain the coefficient of the first derivative of the $l^{-}$distribution as

$$
\begin{aligned}
C_{1} & \propto \frac{-g^{2}}{2 q^{-}} \int d Y^{-} d y^{-}\left\langle p\left|\partial^{-} A^{+}\left(y^{-} / 2\right) \partial^{-} A^{+}\left(-y^{-} / 2\right)\right| p\right\rangle \\
& \simeq \frac{-g^{2}}{2 q^{-}} \int d Y^{-} d y^{-}\left\langle p\left|F^{-+}\left(y^{-} / 2\right) F^{-+}\left(-y^{-} / 2\right)\right| p\right\rangle, \quad
\end{aligned}
$$

where, the standard shorthands of $A^{\alpha}=t^{a} A^{a \alpha}$ and $F^{-+}=$ $t^{a} F^{a-+}$ have been used (trace over color is implied). This term results in a slight gain in the longitudinal momentum fraction. In the derivation above, we have approximated that in the Breit frame (and in a covariant gauge) $A^{+} \gg A_{\perp} \gg A^{-}$[23] 
and $\partial^{-} A^{+} \simeq F^{-+}$. Such terms are only important at lower jet momenta.

In this Rapid Communication, we have presented an extension of the higher-twist expansion formalism of jet modification to include the effect of elastic energy loss. This was carried out by extending the formalism of transverse broadening in Ref. [14], by generalizing the two dimensional distribution of the propagating quark's transverse momentum to a three dimensional distribution including also its longitudinal momentum $l^{-}$. The equation governing the distribution in $l^{-}$includes both a diffusion term and a drag term. In contrast to other formalisms of jet modification, both these terms were evaluated at the operator level, independent of the details of the medium. The sole assumption used was that the color correlation length in the medium is small.
The drag coefficient which yields the elastic energy loss per unit length was then evaluated in a thermal plasma in the HTL limit. The results obtained are consistent with similar calculations in other formalisms [9,10,12]. The final results, however, differed from previous attempts within the higher-twist formalism such as Ref. [11] where elastic energy loss amplitudes interfered with radiative processes. In this Rapid Communication, the focus has been solely restricted to those processes which do not interfere with radiative amplitudes.

The author thanks U. Heinz, B. Müller, and G-Y. Qin for extensive discussions. This work was supported in part by the US Department of Energy, under Grant Nos. DE-FG0205ER41367 and DE-FG02-01ER41190.
[1] M. Gyulassy and M. Plumer, Phys. Lett. B243, 432 (1990); X. N. Wang and M. Gyulassy, Phys. Rev. Lett. 68, 1480 (1992); R. Baier et al., Nucl. Phys. B483, 291 (1997); B484, 265 (1997); B. G. Zakharov, JETP Lett. 65, 615 (1997); Phys. At. Nucl. 61, 838 (1998) [Yad. Fiz. 61, 924 (1998)].

[2] P. Arnold, G. D. Moore, and L. G. Yaffe, J. High Energy Phys. 06 (2002) 030; S. Jeon and G. D. Moore, Phys. Rev. C 71, 034901 (2005); S. Turbide, C. Gale, S. Jeon, and G. D. Moore, ibid. 72, 014906 (2005).

[3] X. F. Guo and X. N. Wang, Phys. Rev. Lett. 85, 3591 (2000); X. N. Wang and X. F. Guo, Nucl. Phys. A696, 788 (2001); B. W. Zhang and X.-N. Wang, ibid. A720, 429 (2003).

[4] M. Gyulassy, P. Levai, and I. Vitev, Phys. Rev. Lett. 85, 5535 (2000); Nucl. Phys. B594, 371 (2001).

[5] U. A. Wiedemann, Nucl. Phys. B588, 303 (2000).

[6] A. Airapetian et al., Eur. Phys. J. C 20, 479 (2001); A. Airapetian et al., Phys. Lett. B577, 37 (2003).

[7] K. Adcox et al., Nucl. Phys. A757, 184 (2005); J. Adams et al. ibid. A757, 102 (2005).

[8] A. Majumder, J. Phys. G 34, S377 (2007).

[9] M. H. Thoma, Phys. Lett. B273, 128 (1991); E. Braaten and M. H. Thoma, Phys. Rev. D 44, R2625 (1991); 44, 1298 (1991).

[10] M. Djordjevic, Phys. Rev. C 74, 064907 (2006); M. G. Mustafa, ibid. 72, 014905 (2005); A. K. Dutt-Mazumder, Jan-e Alam, P. Roy, and B. Sinha, Phys. Rev. D 71, 094016 (2005); S. Wicks et al., Nucl. Phys. A784, 426 (2007).
[11] X. N. Wang, Phys. Lett. B650, 213 (2007).

[12] G. Y. Qin, J. Ruppert, C. Gale, S. Jeon, G. D. Moore, and M. G. Mustafa, Phys. Rev. Lett. 100, 072301 (2008).

[13] J. D. Jackson, Classical Electrodynamics (John Wiley \& Sons, Inc., New York, 1975).

[14] A. Majumder and B. Muller, Phys. Rev. C 77, 054903 (2008); A. Majumder, R. J. Fries, and B. Muller, ibid. 77, 065209 (2008).

[15] E. V. Shuryak and I. Zahed, Phys. Rev. C 70, 021901(R) (2004); Phys. Rev. D 70, 054507 (2004).

[16] V. Koch, A. Majumder, and J. Randrup, Phys. Rev. Lett. 95, 182301 (2005); A. Majumder and B. Muller, Phys. Rev. C 74, 054901 (2006).

[17] X. Guo, Phys. Rev. D 58, 114033 (1998).

[18] A. Majumder and X. N. Wang, arXiv:0806.2653 [nucl-th].

[19] A. Majumder, C. Nonaka, and S. A. Bass, Phys. Rev. C 76, 041902(R) (2007); S. A. Bass et al., Phys. Rev. C 79, 024901 (2009).

[20] J. I. Kapusta and C. Gale, Finite-Temperature Field Theory: Principles and Applications (Cambridge University Press, Cambridge, 2006).

[21] G.-Y. Qin, Ph.D. thesis, McGill University, 2008.

[22] R. B. Neufeld, B. Muller, and J. Ruppert, Phys. Rev. C 78, 041901(R) (2008); R. B. Neufeld, Phys. Rev. D 78, 085015 (2008)

[23] M. Luo, J. Qiu, and G. Sterman, Phys. Lett. B279, 377 (1992); Phys. Rev. D 50, 1951 (1994). 